\title{
COTAS RACIAIS NA UNIVERSIDADE: UMA REVISÃO INTEGRATIVA DA PSICOLOGIA BRASILEIRA
}

\author{
Racial Quotas at the University: An Integrative Review of Brazilian Psychology \\ Cuotas Raciales en la Universidad: Una Revisión Integradora de la Psicología Brasileña
}

Quotas Ethniques a l'Université : Une Revue Intégrative de la Psychologie Brésilienne

doi) $10.5020 / 23590777 . r s . v 20$ iEsp1.e9337

Iara Andrade de Oliveira 9

Mestre em Psicologia pela Universidade de Fortaleza (Unifor). Professora da graduação em Psicologia no Centro Universitário UNIATENEU.

\section{Luciana Maria Maia 9 id}

Doutora em Psicologia Social pela Universidade Federal da Paraíba (UFPB). Professora Titular da Universidade de Fortaleza (Unifor), atuando no Programa de Pós-graduação em Psicologia.

\section{Tiago Jessé Souza Lima 9 id}

Doutor em Psicologia Social pela Universidade Federal da Paraíba. Professor do Departamento de Psicologia Social e do Trabalho e do Programa de Pósgraduação em Psicologia Social, do Trabalho e das Organizações na Universidade de Brasília.

\section{Resumo}

As discussões sobre cotas nas universidades reúnem diversos argumentos, polarizando opiniões sobre sua implantação. Acerca disso, estudos relacionam a oposição às cotas ao racismo e apontam, também, para os usos de argumentos que envolvem as ações afirmativas como forma de legitimar a discriminação contra negros e pobres. Na literatura em psicologia ainda são poucos os estudos que abordam a temática do racismo. Partindo disso, este artigo tem como objetivo caracterizar a produção científica brasileira em psicologia sobre cotas raciais nas universidades. Foi realizada uma revisão integrativa da literatura, na qual foram realizadas buscas nas bases de dados SciELO, PePSIC, Index Psi e LILACS, reunindo 11 artigos. A análise dos artigos identificou lacunas nas descrições dos métodos e pequena variedade nas estratégias de coleta e de análise de dados. A teoria das representações sociais foi a base teórica mais utilizada. Em relação ao conteúdo dos artigos, o corpus foi organizado em dois grandes eixos temáticos: (a) opiniões sobre cotas, com a maioria dos artigos, e (b) vivência de estudantes cotistas. O primeiro grupo de artigos tinha o intuito de conhecer o posicionamento de determinados grupos ou documentos sobre a lei de cotas. O segundo grupo, com apenas dois estudos, buscou conhecer a vivência de estudantes que ingressaram na universidade por meio da lei de cotas. Esses achados, provenientes de um reduzido número de pesquisas sobre a temática, apontam para um campo de estudo que ainda precisa ser explorado, priorizando diferentes estratégias metodológicas na compreensão do fenômeno.

Palavras-chave: cotas raciais; cotas universitárias; racismo; revisão integrativa.

\section{Abstract}

The discussions about quotas in universities bring together several arguments, polarizing opinions about their implementation. About this, studies relate the opposition to quotas to racism and also point to the uses of arguments that involve affirmative action as a way to legitimize discrimination against blacks and the poor. In the literature on psychology, there are still few studies that address racism. Based on this, this article aims to characterize the Brazilian scientific production in psychology on racial quotas in universities. An integrative literature review was carried out, in which searches were carried out in the SciELO, PePSIC, Index Psi and LILACS databases, gathering 11 articles. The analysis of the articles identified gaps in the method descriptions and a small variation in the data collection and analysis strategies. The theory 
of social representations was the most widely used theoretical basis. Regarding the content of the articles, the corpus was organized into two main thematic axes: (a) opinions about quotas, with most articles, and (b) experience of quota students. The first group of articles had the intention of knowing the positioning of certain groups or documents on the quota law. The second group, with only two studies, sought to know the experience of students who entered the university through the quota law. These findings, coming from a small number of researches on the theme, point to a field of study that still needs to be explored, prioritizing different methodological strategies in understanding the phenomenon.

Keywords: racial quotas; university quotas; racism; integrative review.

\section{Resumen}

Las discusiones sobre cuotas en las universidades reúnen diversos argumentos, polarizando opiniones sobre su implementación. Sobre eso, estudios relacionan la oposición a las cuotas al racismo e indican, también, para los usos de argumentos que envuelven las acciones afirmativas como forma de legitimar la discriminación contra negros y pobres. En psicología la literatura es insuficiente en la temática del racismo. Este trabajo tiene el objetivo de caracterizar la producción cientifica brasileña en psicología sobre cuotas raciales en las universidades. Fue realizada una revisión integradora de la literatura, en la cual fueron realizadas búsquedas en las bases de datos SciELO, PePSIC, Index Psi y LILACS, reuniendo 11 artículos. El análisis de los artículos identifico huecos en las descripciones de los métodos y pequeña variedad en las estrategias de colecta y de análisis de datos. La teoría de las representaciones sociales fue la base teórica más utilizada. En relación al contenido de los artículos, el corpus fue ordenado en dos grandes ejes temáticos: (a) opiniones sobre cuotas, con la mayoría de los artículos, y (b) experiencias de estudiantes con cuota. El primer grupo de artículos tenía el objetivo de conocer el posicionamiento de determinados grupos o documentos sobre la ley de cuotas. El segundo grupo, con solo dos estudios, busco conocer la experiencia de Estudiantes que entraron en la universidad por medio de la ley de cuotas. Estos hallazgos, provenientes de un reducido número de investigaciones sobre la temática, indican un campo de estudio que aún necesita ser explorado, priorizando distintas estrategias metodológicas en la comprensión del fenómeno.

Palabras clave: cuotas raciales; cuotas universitarias; racismo; revisión integradora.

\section{Résumé}

Les discussions sur les quotas dans les Universités rassemblent plusieurs arguments, ce qui polarisent les opinions sur leur mise en cuvre. À ce sujet, des études lient l'opposition aux quotas au racisme. Ils soulignent, également, l'utilisation d'arguments qui impliquent une action positive comme moyen de légitimer la discrimination contre les Noirs et les pauvres. Dans la littérature sur la psychologie, il y a encore peu d'études qui traitent du thème du racisme. Sur cette base, cet article vise à caractériser la production scientifique brésilienne en psychologie sur les quotas ethniques dans les universités. Une revue de littérature intégrative a été réalisée, dans laquelle des recherches ont été effectuées dans les bases de données SciELO, PePSIC, Index Psi et LILACS, regroupant 11 articles. L'analyse des articles a identifié des lacunes dans les descriptions des méthodes et une petite variété dans les stratégies de collecte et d'analyse des données. La théorie des représentations sociales a été la base théorique la plus largement utilisée. Concernant le contenu des articles, le corpus a été organisé en deux axes thématiques principaux: (a) opinion sur les quotas, avec la plupart des articles, et (b) expérience des étudiants en quota. Le premier groupe d'articles avait l'intention de connaître le positionnement de certains groupes ou documents sur la loi sur les quotas. Le seconde groupe, avec seulement deux études, a essayé connaître l'expérience des étudiants qui sont entrés à l'université à travers de la loi des quotas. Ces résultats, venus d'un petit nombre de recherches sur le thème, pointent vers un champ d'étude qui reste à explorer, privilégiant différentes stratégies méthodologiques pour comprendre le phénomène.

Mots-clés : quotas ethniques ; quotas universitaires ; racisme ; revue intégrative.

O Brasil é um país que, historicamente, apresenta altas e persistentes desigualdades. A Pesquisa Nacional por Amostra de Domicílios (PNAD) de 2015, realizada pelo Instituto Brasileiro de Geografia e Estatística [IBGE], revelou que apenas $15 \%$ dos domicílios brasileiros possuíam renda per capita maior que dois salários mínimos. Em relação à escolaridade, nesse mesmo período, $52 \%$ da população de 25 anos ou mais tinha cursado até o ensino fundamental completo, enquanto $13,5 \%$ possuíam o superior completo.

Toma-se aqui o conceito de raça a partir da consideração de que, embora não existam diferenças raciais entre seres humanos, existe uma segregação e hierarquização racial em termos sociais e culturais (Carvalho, 2014). Se realizado um recorte de raça, os dados revelam que a população negra representa $74 \%$ das pessoas com menores rendimentos do país 
(IBGE, 2016). Além disso, resultados de 2012 revelam que 22,2\% da população branca e 9,4\% da negra estudavam 12 anos ou mais (Instituto de Pesquisa Económica Aplicada [IPEA], 2014).

Reconhecendo essas disparidades econômicas e raciais no Brasil, diferentes medidas no âmbito jurídico têm sido tomadas nas últimas décadas, sobretudo durante os governos do presidente Lula (2003 - 2011). Nesse contexto, os processos de implementação das políticas de cotas nas instituições públicas de ensino superior vêm mobilizando o debate público e a produção acadêmica (Lima, 2010). Mesmo antes da lei federal que instituiu as cotas nas universidades ser sancionada, o governo federal já sinalizava apoio explícito às ações afirmativas. Assim, o processo de cotas para negros e índios iniciou sendo adotado individualmente pelas universidades federais e estaduais.

Em 2003, a Universidade Estadual do Rio de Janeiro (Uerj) implementou o primeiro programa de cotas brasileiro (Guarnieri \& Melo-Silva, 2017). Em 2004, a Universidade de Brasília (UNB) adotou a política de cotas étnico-raciais, sendo a primeira instituição federal do gênero a adotar tal ação (Lima, Neves, \& Silva, 2014). Em 2010, 91 instituições públicas de ensino de nível superior já adotavam algum tipo de política afirmativa para ingresso desses grupos nos cursos de graduação (Lima, 2010).

Desde que a primeira instituição federal de ensino superior adotou cotas em seu processo seletivo, o debate público sobre a política de cotas tem sido recorrente. Em 2010 foi proposta uma arguição de descumprimento de preceito fundamental contra as cotas universitárias criadas na UNB. Essa arguição alegava que as cotas violariam os preceitos constitucionais do princípio da dignidade humana, do repúdio ao racismo, da igualdade, direito universal a educação e da meritocracia. Todavia o Supremo Tribunal Federal (STF), julgou em unanimidade o pedido como improcedente (Schulze, 2012).

Somente oito anos depois da UNB ter adotado em seu processo seletivo as cotas universitárias, o Governo Federal, em agosto de 2012, instituiu a chamada Lei de Cotas para o Ensino Superior (Lei n. 12.711, 2012). A medida, que provocou muitas críticas, foi sancionada com o objetivo de diminuir os efeitos da desigualdade de classe e racial, por meio da reserva de vagas em instituições federais de ensino superior. De acordo com essa lei, cada concurso seletivo para ingresso nos cursos de graduação, por curso e turno em instituições federais, passa a ter, no mínimo, $50 \%$ das vagas para estudantes que tenham cursado integralmente o ensino médio em escolas públicas e, destes, $50 \%$ são reservados aos estudantes oriundos de famílias com renda igual ou inferior a 1,5 salários-mínimos per capita. Dentro desses requisitos, a proporção de autodeclarados negros (pretos e pardos) e indígenas deve ser, no mínimo, igual à proporção respectiva desses grupos na população da unidade da federação onde se localiza a instituição. A lei também instituiu que, no prazo de 10 anos, ou seja, em 2022, será promovida uma revisão de seu conteúdo.

Mesmo após a promulgação da lei federal, os debates em relação às cotas universitárias ainda persistem. No contexto da internet, as discussões sobre o tema não apresentam elementos consensuais sobre a validade da implantação de cotas (Castro, Koelzer, Camargo, \& Bousfield, 2014). Estudos que investigaram a opinião de universitários sobre o tema também revelam posições variadas, embora os estudantes, em sua maioria, tenham sido contrários às cotas sociais e, principalmente, às raciais (Lima et al., 2014). Um estudo que analisou artigos sobre cotas publicados de 2008 a 2012 evidenciou que existe uma nova forma de operacionalização do racismo, que utiliza as cotas como justificativa (Cruz, 2016). Já um estudo experimental, com estudantes do ensino médio, detectou uma menor avaliação positiva de pessoas cotistas, independentemente da cor de pele (Faro, Silva, \& Machado, 2016).

Desse modo, é possível perceber a relação entre as temáticas do racismo com cotas raciais universitárias. Embora a segregação racial nunca tenha sido oficializada por lei no Brasil, o racismo é perceptível nas ruas, nos espaços públicos e privados, como bem ilustraram os dados estatísticos apresentados anteriormente. A despeito da relevância da temática, os estudos desenvolvidos sobre o racismo por pesquisadores da psicologia no Brasil ainda são escassos, sendo possível perceber uma diferença quantitativa em relação às produções sobre essa temática em outras áreas das ciências sociais, a exemplo da sociologia e da antropologia (Munanga, 2012).

Em uma revisão sistemática da literatura da psicologia brasileira sobre preconceito racial (Sacco, Couto, \& Koller, 2016), verificou-se, ainda, um número relativamente baixo de publicações sobre o tema. A revisão foi realizada nas bases SciELO, PePSIC, Index Psi e LILACS sobre os temas racismo e preconceito racial. A pesquisa incluiu 77 artigos publicados por pesquisadores brasileiros da área, no período de 2001 a 2014, uma quantidade de publicação considerada baixa para um período de 13 anos. Além disso, a maioria das pesquisas foi conduzida com estudantes universitários, sendo poucas as que tiveram a participação de adultos não universitários e pessoas que pertençam ao grupo discriminado. Diante desses aspectos, as autoras ressaltaram que os estudos nacionais ainda estão pouco inseridos em relação às discussões de outros países. Desse modo, o tema racismo ainda está longe de ser saturado entre os assuntos investigados pelos pesquisadores brasileiros na área da psicologia.

Perante essa temática, realizar estudos sobre cotas raciais na universidade também implica discutir e compreender como o racismo se apresenta no Brasil. Sendo assim, as discussões sobre essas cotas podem ser um elemento de reconhecimento desse problema. Diante do exposto, esse trabalho tem como objetivo caracterizar a produção científica brasileira no campo da psicologia sobre cotas raciais na universidade, buscando compreender o que os pesquisadores brasileiros dessa área têm estudado sobre os processos que envolvem as cotas raciais nas instituições de ensino superior. 


\section{Método}

\section{Tipo de Estudo}

Essa pesquisa é caracterizada como uma revisão integrativa da literatura, tendo como característica principal reunir e sintetizar resultados de pesquisa sobre um tema através de procedimentos de coleta e análise de textos, realizados de forma sistemática e organizada. Foram seguidos os procedimentos propostos por Mendes, Silveira, e Galvão (2008): (1) identificar o tema e seleção da hipótese ou questão de pesquisa; (2) estabelecer critérios para inclusão e exclusão dos estudos e busca na literatura; (3) extrair informação e categorização dos estudos; (4) avaliar os estudos; (5) interpretar os resultados; e (6) sintetizar o conhecimento. A questão norteadora deste estudo foi: o que tem sido produzido na psicologia brasileira sobre as cotas raciais na universidade?

\section{Bases de Dados e Termos de Busca}

As bases de dados utilizados para a investigação desta revisão foram a SciELO, PePSIC, Index Psi e LILACS. Essas bases foram selecionadas por concentrarem grande parte da produção científica nacional na área em foco. Ademais, são bases que proporcionam o acesso público e gratuito aos artigos, constituindo um veículo relevante para a divulgação das produções científicas. Os termos de busca com operadores booleanos utilizados foram: "cotas raciais $O R$ cotas universitárias", nas três primeiras bases acima mencionadas, e "(racial quotas OR university quotas) AND (Brazil OR Brazilian AND portuguese)" na base LILACS.

\section{Critérios de Inclusão e Exclusão}

Foram incluídos os artigos que se encaixaram nos seguintes critérios: (1) artigos que abordem o tema cotas raciais na universidade; (2) artigos publicados em periódicos brasileiros da psicologia. Não foi estabelecida uma delimitação do período de publicação. Foram excluídos os artigos que se encaixaram nos critérios a seguir: (1) os artigos duplicados entre as bases consultadas; (2) artigos que apenas mencionavam as cotas raciais, sem tratar dessa temática em seus objetivos ou resultados; (3) artigos que não foram publicados em periódicos brasileiros da psicologia; (4) artigos que não possuíam texto completo disponível na internet; e (5) artigos de revisão sistemática da literatura ou artigos teóricos sobre o tema.

\section{Coleta de Dados}

Os levantamentos foram realizados em um único dia, 3 de julho de 2018. Todo o processo de seleção dos artigos foi executado por duas juízas, que realizaram esse trabalho de forma separada e, em um segundo momento, verificaram os critérios de inclusão e exclusão em conjunto.

\section{Análise dos Dados}

Os artigos selecionados foram catalogados em uma planilha de Excel composta pelas colunas: título, autores, palavraschave, ano de publicação, data de envio, data de aceite, periódico, qualis, objetivo, base teórica, método e resultados. A análise dos dados foi feita a partir dessas categorias. Partindo de uma análise mais detalhada do corpus, com base no tema do estudo, base teórica, método e resultados encontrados, e para melhor compreensão dos achados, o material foi organizado em dois eixos temáticos: (a) opiniões sobre cotas e (b) vivência de estudantes cotistas.

\section{Resultados e Discussão}

A busca inicial nas bases de dados gerou um total de 36 artigos (SciELO: 11, PePSIC: 1, Index Psi: 6, LILACS: 18). $\mathrm{Na}$ primeira triagem, foram excluídos oito trabalhos duplicados entre as bases. Dos 28 artigos restantes, dois atenderam ao segundo critério de exclusão, já que os textos não se referiam diretamente ao tema das cotas raciais universitárias, citando a temática apenas para contextualizar outra questão. Dos 26 restantes, 12 não tinham sido publicados em periódicos de psicologia, um não possuía texto completo disponível na internet, um era um estudo de "estado da arte" e um era um estudo teórico. Assim, restaram 11 artigos (ver Figura 1), destacados com asterisco na lista de referências, que compuseram o corpus do presente estudo de revisão, correspondendo a 30,55\% dos estudos encontrados nas buscas. 


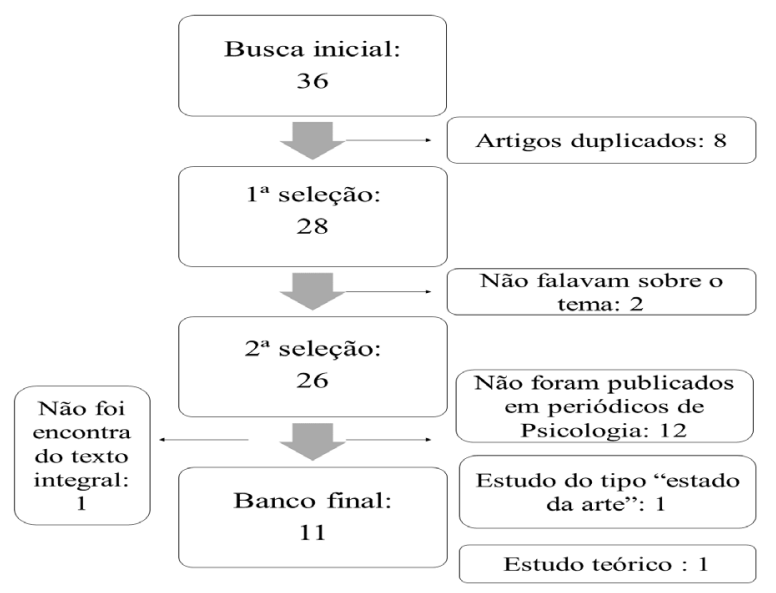

Figura 1. Fluxograma de seleção de artigos.

O corpus analisado nesta pesquisa está publicado em sete revistas diferentes (ver Tabela 1). As revistas que mais se destacaram foram Psicologia: Ciência e Profissão (2), Psicologia e Saber Social (2) e Psicologia \& Sociedade (3). As três revistas propõem-se a discutir temas socialmente relevantes e que se relacionem com a Psicologia, sendo uma possível explicação para serem as revistas que mais publicaram sobre cotas raciais na universidade.

Tabela 1.

Número de artigos publicados por revista

\begin{tabular}{lcl}
\hline \multicolumn{1}{c}{ Revista (Qualis) } & Quantidade & \multicolumn{1}{c}{ Referência } \\
\hline Psicologia Politica (B2) & 1 & Mayorga \& Souza (2012) \\
Psicologia e Saber Social (B2) & 2 & Melo, Dantas, Fernandez, Pereira, \& Chaves (2014); Marques \& Santos (2015). \\
Estudos e pesquisas em Psicologia (B1) & 1 & Naiff, Naiff, \& Souza (2009) \\
Fractal: Revista de Psicologia (B1) & 1 & Silva \& Silva (2012) \\
Estudos de Psicologia (A2) & 1 & Oliveira Filho (2009) \\
& 3 & Guarnieri \& Melo-Silva (2010); Camino, Gouveia, Maia, Paz, \& Laureano \\
Psicologia \& Sociedade (A2) & $2013) ;$ Camino, Tavares, Torres. Álvaro, \& Garrido (2014). \\
Psicologia: Ciência e Profissão (A2) & 2 & Ferreira \& Mattos (2007); Jesus (2013). \\
\hline
\end{tabular}

Em relação ao qualis das revistas, de acordo com o qualis de periódicos da Coordenação de Aperfeiçoamento de Pessoas de Nível Superior (Capes) no período de referência 2013/2016, 42,8\% (N = 3) das revistas aqui apresentadas foram classificadas no estrato $\mathrm{A}$, sendo todas $\mathrm{A} 2$, e $57,1 \%(\mathrm{~N}=4)$ foram classificadas no estrato $\mathrm{B}$, sendo duas $\mathrm{B} 1$ e duas $\mathrm{B} 2$. Em relação ao quantitativo de artigos $54,5 \%(\mathrm{~N}=6)$, estão em revistas do segmento A. Assim, embora a quantidade de artigos publicados ainda seja restrita, considerando os critérios de inclusão e exclusão adotados para esta revisão integrativa, a qualidade das revistas que publicaram os artigos que integram o corpus sinaliza a qualidade dos artigos e a relevância dessa temática.

Ao analisarmos as datas de publicação dos artigos encontrados, observamos que as publicações sobre esse tema nos periódicos da psicologia tiveram início em 2007 (ver Figura 2). Observando-se a existência de publicações por ano, pode-se notar que, após 2012, ano no qual a lei de cotas foi sancionada, a frequência das publicações sobre essa temática tornou-se mais contínua. Considerando-se o período de 2007 até 2012, observa-se que não existiram publicações sobre o tema nos anos de 2008 e 2011. Já no período de 2012 até 2016, observa-se que a temática foi recorrente em todos os anos. 


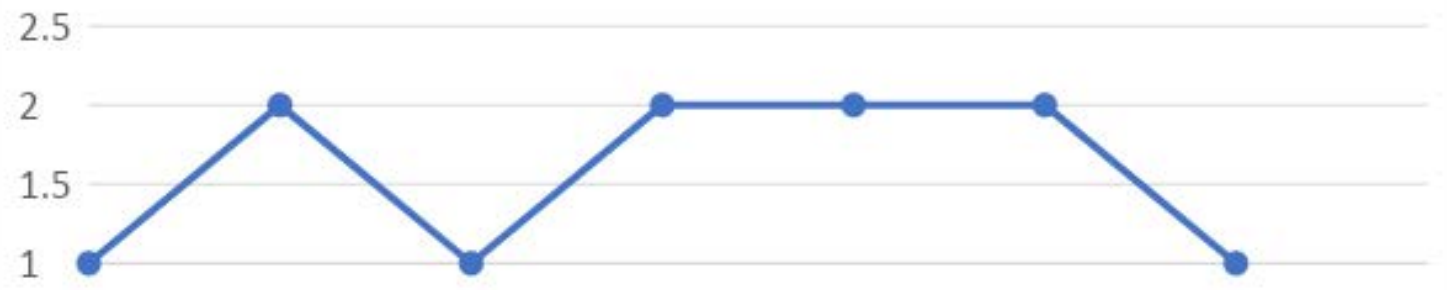

1

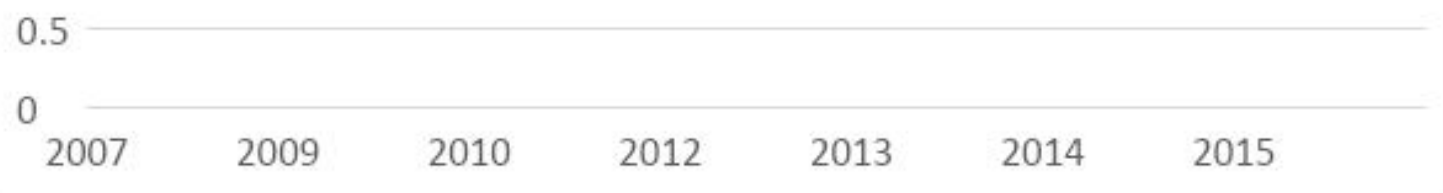

Figura 2. Número de artigos publicados por ano.

Observando os dados dos artigos de maneira mais atenta, analisando as datas de envio e aceite dos periódicos, notase que, entre os 11 artigos aqui encontrados, $81,8 \%(\mathrm{~N}=9)$ foram enviados antes da promulgação da lei e apenas $18,2 \%$ $(\mathrm{N}=2)$ foram enviados depois. Dessa forma, embora a quantidade de artigos encontrados não permita concluir nenhum tipo de tendência temporal sobre as publicações, é possível notar que, após o ano da promulgação da lei de cotas, 2012, as publicações sobre o tema passaram a ter a frequência de pelo menos um artigo ao ano, porém nota-se que somente uma quantidade reduzida de artigos, apenas dois, foi realmente enviada após a promulgação da lei.

Sendo assim, essas informações apontam que a temática já vinha sendo discutida mesmo antes da promulgação da lei federal, quando as cotas ainda não eram implementadas em todo o país. Podendo indicar, também, que, após a promulgação da lei, as revistas tiveram mais interesse em realizar publicações sobre o assunto. Nenhuma produção que obedecesse aos critérios desta revisão, entretanto, foi encontrada no período de janeiro de 2016 a 3 de julho de 2018, o que pode indicar um desinteresse pela temática passados três anos da promulgação da lei de cotas.

Com relação à base teórica dos estudos, a psicologia social foi a mais utilizada, sendo que, de maneira específica, 63,6\% $(\mathrm{N}=7)$ utilizaram como aporte a teoria das representações socais. Outro aspecto comum entre os estudos foi o de avaliar ou analisar o posicionamento existente sobre cotas na universidade.

Em relação ao método dos artigos, foram encontradas algumas lacunas nas descrições das informações que deveriam compor tal seção. Em três estudos (Camino, Gouveia, Maia, Paz, \& Laureano, 2013; Camino, Tavares, Torres, Álvaro, \& Garrido, 2014; Melo, Dantas, Fernandez, Pereira, \& Chaves, 2014) os dados sociodemográficos dos participantes não estavam descritos de maneira a identificar percentuais de pessoas negras ou cotistas que responderam à pesquisa. Considerando a temática das cotas raciais e os estudos sobre racismo e preconceito, a informação sobre a identidade étnicoracial dos participantes é de extrema relevância, tendo em vista que essa identidade pode afetar os resultados das pesquisas. A falta dessa informação pode levar a conclusões enganosas; correndo o risco, inclusive, de contribuir para uma perspectiva colonialista das temáticas (Lima, 2014).

Outros estudos não apresentaram seus métodos de maneira explícita, como foi o caso da revisão documental realizada por Ferreira e Mattos (2007) e da pesquisa intervenção de Mayorga e Souza (2012). A presença de lacunas na descrição do método de pesquisa ou a ausência dessa informação no artigo podem limitar o desenvolvimento da ciência. A descrição do método, além de facilitar a replicação de estudos futuros, também auxilia na compreensão dos resultados, tendo em vista que os participantes da pesquisa, a ferramenta de coleta utilizada e a maneira como os dados foram analisados podem alterar os apontamentos dos estudos (Creswell, 2007).

Em relação ao tipo de método utilizado, $81,8 \%(\mathrm{~N}=9)$ utilizaram métodos qualitativos de coletas de dados e 18,2\% (N =2) métodos mistos. Nenhum dos estudos possuiu enfoque apenas quantitativo. Em relação à análise de dados, em $27,3 \%$ $(\mathrm{N}=3)$ dos artigos utilizaram a análise de conteúdo (Guarnieri \& Melo-Silva, 2010; Marques \& Santos, 2015; Silva \& Silva, 2012) e em 54,5\% ( $=6$ ) dos estudos utilizaram algum tipo de software, a exemplo do EVOC (Jesus, 2013; Melo et al., 2014; Naiff, Naiff, \& Souza, 2009) e do Alceste (Camino et al., 2013; Camino et al., 2014; Melo et al., 2014). O aporte teórico da psicologia social discursiva foi utilizado para a análise dos dados de um estudo (Oliveira, 2009) e outro realizou uma pesquisa intervenção (Mayorga \& Souza, 2012). 
Sobre os instrumentos utilizados, 36,4\% $(\mathrm{N}=4)$ realizaram entrevistas, $36,4 \%(\mathrm{~N}=4)$ utilizaram associação livre de palavras e um estudo utilizou grupo focal (Silva \& Silva, 2012). Entre os estudos com método misto, um realizou entrevistas e aplicação de um questionário (Guarnieri \& Melo-Silva, 2010) e outro utilizou o método de associação livre de palavras e um questionário com perguntas abertas (Melo et al., 2014).

Desse modo, tendo em vista as informações supracitadas, não se percebe uma grande variabilidade de métodos nesse corpus. Nenhum dos estudos encontrados apresentou delineamento experimental ou quasi-experimental, estratégia muito utilizada fora do Brasil, semelhante ao que Sacco et al. (2016) sinalizam em uma revisão sobre o tema do racismo na psicologia brasileira. Os delineamentos experimental e quasi-experimental têm como objetivo apresentar maior controle sobre variáveis envolvidas e possibilitar acesso ao conteúdo que as regras sociais não permitem que sejam expressos de maneira explicita.

Em relação ao conteúdo dos artigos, observa-se que nove têm como foco principal investigar posicionamentos e percepções acerca das cotas raciais nas universidades. Partindo dessa observação, o conteúdo do corpus da pesquisa foi organizado em dois grandes eixos temáticos: (a) opiniões sobre cotas e (b) vivência de estudantes cotistas.

O eixo opiniões sobre cotas foi composto por artigos que buscam compreender quais as opiniões que circulam sobre as cotas raciais universitárias (Camino et al., 2013; Camino et al., 2014; Ferreira \& Mattos, 2007; Guarnieri \& Melo-Silva, 2010; Marques \& Santos, 2015; Melo et al., 2014; Naiff et al., 2009; Oliveira, 2009; Silva \& Silva, 2012). O eixo vivência dos estudantes cotistas é composto por pesquisas realizadas com estudantes que entraram na universidade por cotas e abordam as experiências desses estudantes no contexto universitário (Jesus, 2013; Mayorga \& Souza, 2012).

\section{Opiniões sobre Cotas}

As pesquisas que investigaram a opinião sobre cotas, de maneira geral, apresentam resultados nos quais os critérios sociais para as cotas são mais bem aceitos que os raciais.

Os resultados das pesquisas parecem indicar, também, que os participantes não possuem conhecimento total sobre o conteúdo da lei de cotas, tendo em vista que os critérios sociais também se aplicam às cotas raciais.

Esses resultados aparecem em amostras de variados contextos de pesquisa, a exemplo de universidades que já haviam adotado a cota antes mesmo da promulgação da lei federal, como a Universidade Federal da Bahia (UFBA; Silva \& Silva, 2012) e a Universidade Federal da Paraíba (UFPB; Camino et al., 2013; Camino et al., 2014), ou cogitavam adotar, caso da Universidade Federal Rural do Rio de Janeiro (UFRRJ; Naiff et al., 2009), e em pesquisas realizadas com a população geral (Oliveira, 2009), com estudantes pré-universitários (Guarnieri \& Melo-Silva, 2010) e na impressa escrita (Ferreira \& Mattos, 2007). Analisando as publicações antes e após a promulgação da lei de cotas, observa-se que esses padrões de resultados se mantêm, mesmo com a lei já implementada, como ilustram os dois artigos que foram produzidos após a promulgação da lei (Marques \& Santos, 2015; Melo et al., 2014).

Entre os argumentos que configuram as justificativas relatadas nos estudos sobre os posicionamentos contra a implementação da lei de cotas, estão: (1) a importância do mérito, (2) a possível redução do nível acadêmico e (3) a problematização de que o governo deveria, primeiro, melhorar a educação de base, antes de realizar ações como essas. Especificamente sobre o critério étnico-racial da lei de cotas, revelam-se argumentos sobre: (1) a dificuldade de definir quem seria negro no Brasil devido à miscigenação, (2) a percepção de que o problema da desigualdade no acesso à universidade seria social e não racial e (3) a existência de uma democracia racial no Brasil. Essas justificativas que se assemelham às dadas pelo partido dos Democratas (DEM), ainda em 2010, quando solicitava ao STF a revogação da decisão administrava da UNB na adoção de cotas (Schulze, 2012).

Em relação aos três principais argumentos sobre a não aceitação das cotas sociais, é possível percebê-los como formas de legitimar a discriminação e manter o status quo. Sobre a importância do mérito, estudos mostram que os valores igualitários estruturam a norma antipreconceito; já valores protestantes, como o da meritocracia, podem ser associados ao preconceito e a discriminação, pois facilitam a legitimação para tais atitudes e comportamentos. Quando os valores meritocráticos estão salientes, as relações entre preconceito e discriminação ocorrem de forma mais direta, não necessitando de justificativa ou sustentando a justificativa no próprio valor de mérito (Pereira \& Vala, 2011).

A respeito do argumento sobre a provável redução do nível acadêmico nas universidades, é possível pensar em dois processos diferentes de legitimação da discriminação. O primeiro pode ser relacionado ao preconceito de forma direta, pois esse argumento persiste mesmo com diferentes estudos e notícias mostrando que a diferença do desempenho entre estudantes que entraram na universidade com e sem cotas é muito pequena. Portanto, embora existam evidências favoráveis aos cotistas, as crenças e expectativas negativas continuam sendo um elemento comum ao preconceito destinado ao grupo social (Lima, 2013).

Por outro lado, é possível perceber esse argumento como a percepção da existência de uma ameaça real e simbólica (Pereira \& Vala, 2011) para as instituições universitárias e para o desenvolvimento do conhecimento. No caso, a ameaça real seria a de que o exogrupo, formado por estudantes cotistas, estaria ameaçando os interesses do endogrupo, formado por 
estudantes não cotistas. A ameaça simbólica seria relacionada à identidade dos universitários que poderia ser afetada devido à redução do nível acadêmico e pela presença de pessoas de outras etnias e classes socais na instituição de ensino superior.

Isso é apresentado por Camino et al. (2014) em um estudo que compara achados de duas pesquisas. Na primeira, as cotas ainda não tinham sido implementadas na universidade e os resultados indicaram discursos claramente favoráveis às cotas raciais para instituições públicas de ensino superior (Duarte, 2008). Na segunda pesquisa, realizada na mesma universidade, após a implementação das cotas raciais, esses discursos a favor das cotas não apareceram (Camino et al., 2014). Assim, quando a ameaça do status quo se torna real, o posicionamento dos participantes contra essa mudança aparece de maneira mais explícita. Esses achados vão na direção de resultados de estudos que tratam da relação entre preconceito, normas sociais e legitimação (Pereira \& Souza, 2016; Pereira \& Vala, 2011).

$\mathrm{O}$ argumento de que o governo deveria melhorar a educação de base antes de realizar ações como as cotas universitárias pode ser analisado de forma paradoxal. Embora se apresente como uma justificativa de contraposição às cotas universitárias, pode-se considerar a inexistência de igualdade para competir por uma vaga na universidade entre estudantes de escolas públicas e particulares, porém, ao mesmo tempo, essa argumentação ainda se sustenta pela ideologia meritocrática, pois, teoricamente, se o governo melhorar a educação de base, as pessoas irão conseguir entrar na universidade por mérito próprio, e a conjuntura socioeconômica dessas pessoas não precisaria mais ser considerada como fator de desigualdade para esse acesso.

Em relação aos argumentos especificamente contrários ao critério étnico-racial da lei de cotas, parecem indicar a negação da existência de discriminação racial no Brasil, embora se reconheça as disparidades sociais entre brancos e negros. Tal aspecto pode estar relacionado com a negação do racismo dos próprios respondentes, que negam ser racistas, e apontam que existe racismo no Brasil, mas diante do posicionamento de uma ação afirmativa que busca reparar as disparidades raciais do país, mostram-se contrários (Lima, 2002; Lima \& Vala, 2004). Assim, para justificar a não aceitação das cotas raciais, as pessoas utilizam o argumento de que o problema seria de classe (Lima, Pereira, Torres, Souza, \& Albuquerque, 2019), contudo também não aceitam a cota social usando justificativas baseadas na defesa da meritocracia e no não reconhecimento, em suas análises, do critério de classe existente nas cotas raciais.

Esses argumentos podem ser discutidos à luz do mito da democracia racial, ilustrado por Freyre (1933/2017), em Casa Grande e Senzala, sendo outra forma de legitimar a discriminação e o preconceito. De fato, se o Brasil vivesse em uma democracia racial, não existiriam motivos para a implementação de cotas. Embora, estruturalmente, perceba-se que a maioria das pessoas em situações de vulnerabilidade, com menor renda e menor nível de escolaridade, são negras, esses problemas são justificados como uma questão social e não racial (Lima, 2013; Pereira \& Vala, 2011). O argumento de que existiria dificuldade em definir quem seria negro no Brasil devido à miscigenação também pode ser discutido a partir da perspectiva de Freyre (1933/2017). De acordo com o autor, a miscigenação da população brasileira seria uma prova da boa convivência entre brancos e negros, negando violências e abusos dos brancos com os negros.

Outra forma de legitimar o preconceito é direcioná-lo para outro alvo. Tendo em vista que as normas sociais se opõem a formas explicitas de racismo, a discriminação seria deslocada para outro grupo, de modo que não seriam mais os negros os alvos, mas, sim, os cotistas (Cruz, 2016). Essa discussão pode ser ampliada considerando estudos que buscaram conhecer a vivência de estudantes cotistas nas universidades, que serão apresentados na sessão a seguir.

\section{Vivência de Estudantes Cotistas}

Mayorga e Souza (2012) realizaram sua pesquisa com base em informações de estudantes que ingressaram na Universidade Federal de Minas Gerais (UFMG) por meio de cotas, buscando compreender a trajetória desses alunos e como as políticas de permanência contribuem para que eles continuem cursando o ensino superior. As autoras realizaram o recorte desse aspecto da realidade tendo em vista que é comum entre os opositores das políticas afirmativas apontarem como problema para sua implementação na universidade a queda na qualidade de ensino e as dificuldades que serão encontradas por esses alunos no percurso acadêmico. Assim, buscaram trazer as políticas de ação afirmativa de permanência como centro da reflexão. As autoras apresentam a importância dessa ação para os alunos que ingressaram na universidade por cotas continuarem seus cursos universitários e também aspectos relativos à vivência desses estudantes na universidade. Esse assunto parece ser pouco discutido entre as produções científicas da psicologia brasileira, como observado no corpus que está sendo analisado nesta revisão integrativa.

Estudos de outras áreas do conhecimento que investigaram o percurso de estudantes cotistas apontam que esses perduram mais nos estudos, apresentando menor evasão e maiores taxas de graduação (Bezerra \& Gurgel, 2011; Mendes, 2014; Velloso, 2009). Em relação ao desempenho, não se encontra unanimidade entre os estudos, existindo resultados de melhor desempenho dos não cotistas (Mendes, 2014) e de semelhança no desempenho, sem diferenças expressivas, (Bezerra \& Gurgel, 2011), podendo até existir melhor desempenho dos cotistas em determinadas situações (Velloso, 2009). A diferença entre os resultados apresentados nesses estudos pode estar relacionada ao contexto, período e indicadores utilizados nas pesquisas. 
Outro estudo encontrado nessa revisão, que foi realizado com estudantes cotistas, foi desenvolvido por Jesus (2013). A autora apresenta um relato de experiência perpassado por uma descrição breve de um estudo empírico, em que mostra estratégias utilizadas na UNB para ampliar os espaços de acolhimento de estudantes oriundos do Sistema de Cotas para Negros, bem como resultados de uma pesquisa de opinião desses estudantes sobre o assunto. Como estratégia, tem-se o Centro de Convivência Negro (CCN), uma demanda da Assessoria de Diversidade e Apoio aos Cotistas (ADAC), que consiste em uma sala de apoio ao grupo de estudantes que ingressaram na universidade por meio de cotas raciais e é aberta a toda a comunidade acadêmica com o intuito de sensibilizar sobre o tema da negritude e da diversidade. Os resultados apontaram que os usuários do espaço o consideram como local de acolhida e combate ao preconceito existente no campus. A autora sinaliza a importância de divulgar experiências como essa tendo em vista os processos de subjetivação dos afrobrasileiros nesses espaços.

Somente esses dois estudos encontrados buscaram conhecer a perspectiva de estudantes que ingressaram na universidade por meio da lei de cotas, sendo realizados exclusivamente com pessoas pertencentes a esse grupo. Esse resultado assemelhase ao apresento por Sacco et al. (2016) em uma revisão sistemática sobre os estudos com foco em preconceito racial publicados pela psicologia brasileira. As autoras apontaram que poucos estudos têm sido realizados com foco nas pessoas que pertencem ao grupo discriminado. Ademais, Lima (2014) aponta a escassez de estudos na psicologia social com adultos não universitários, tendo em vista que existe um uso excessivo de pesquisas com amostras compostas por estudantes universitários, que, em geral, são de classe média ou alta, ocidentais e brancos.

\section{Considerações Finais}

Essa revisão integrativa teve como objetivo caracterizar a produção científica brasileira em Psicologia sobre cotas raciais na universidade. Observou-se que os periódicos científicos que mais publicaram sobre esse tema foram aqueles com preocupações voltadas à interface da Psicologia com a sociedade, os quais foram avaliados positivamente de acordo com o qualis da Capes, o que pode sinalizar a qualidade das produções sobre cotas raciais no Brasil. Foi encontrado, contudo, um baixo número de pesquisas, lacunas metodológicas e pouca variabilidade de métodos e objetivos nas pesquisas.

Os estudos, em sua maioria, expuseram a opinião de pessoas acerca das cotas universitárias. Em relação às cotas sociais, fala-se na importância da melhoria do ensino básico e das conquistas por mérito, desconsiderando-se o contexto social em que essas pessoas estão inseridas. Em relação às cotas raciais, embora os participantes concordem que existem disparidades raciais no país, eles discordam da reserva de vagas, tendo como um dos argumentos a existência de uma disparidade social, não racial. Assim, para se justificar a não aceitação das cotas raciais, utiliza-se o argumento do problema ser uma questão social, demonstrando o desconhecimento de muitas pessoas sobre a lei, pois as cotas raciais também são sociais. Mas, além disso, há uma rejeição da cota social devido à importância dada aos argumentos de base meritocrática.

A maioria dos artigos utilizou a teoria das representações socais como aporte. Embora o conhecimento sobre as representações que existem em volta dessa temática sejam importantes para que se compreendam as relações sociais e situações de discriminação existentes no Brasil, é necessário que os estudos sobre as cotas sejam aprofundados, que se desenvolvam pesquisas que investiguem como as pessoas se comportam em relação a esse assunto e quais as consequências dessas representações de maneira prática. Dessa forma, pode-se pensar nas potencialidades de se desenvolver pesquisas que abordem outros objetivos, para além de conhecer o posicionamento dos participantes sobre a aceitação ou não de cotas na universidade.

Além disso, nenhuma produção que obedecesse aos critérios desta revisão foi encontrada após 2015, o que pode indicar uma redução ainda maior dos estudos na psicologia brasileira sobre cotas ao passar dos anos em que a lei federal sobre o assunto foi promulgada. Esse dado merece atenção tendo em vista que, em um prazo de 10 anos desde que a lei foi promulgada, ou seja, em 2022, será promovida uma revisão do seu conteúdo. Embora se percebam mudanças significativas, os dados ainda indicam que a desigualdade entre brancos e negros é um grave problema social. Desse modo, é necessário continuar investigando os reflexos das cotas e o que as pessoas pensam sobre o tema.

Os achados encontrados nessa revisão revelam que essa temática ainda pode ser bastante explorada na psicologia brasileira. O tema poderia ser abordado com variações, tanto de referenciais teóricos como de perspectivas metodológicas, a exemplo de pesquisas experimentais ou quasi-experimentais. Os achados dessa revisão se aproximam de estudos que investigam a produção da psicologia brasileira sobre a temática do racismo. Analisando o campo mais amplo de investigação sobre a temática, o escasso número de produções na psicologia pode ser relacionado à dificuldade dos profissionais da área em reconhecer o racismo como problema de investigação científica.

Embora se faça essa consideração, é relevante destacar que esta revisão integrativa se restringiu a artigos científicos publicados na internet, não incluindo teses, dissertações e livros sobre o tema. Além disso, é possível que os descritores "cotas raciais" e "cotas universitárias" não tenham permitido alcançar trabalhos que utilizam nomenclaturas mais generalistas para abordar o assunto das cotas raciais, tais como "ações afirmativas" ou "políticas afirmativas". 
Apesar disso, ressalta-se a importância dos resultados aqui apresentados enquanto um recorte da produção de conhecimento sobre o tema. Desse modo, discutiu-se o enfoque dado pelos estudos na psicologia brasileira, sinalizando perspectivas teóricas e metodológicas que ainda não foram utilizadas tendo em vista o desenvolvimento das investigações no campo do racismo.

\section{Referências}

Bezerra, T., \& Gurgel, C. (2011). A política pública de cotas em universidades, desempenho acadêmico e inclusão social. SBIJ, 09, 1-22.

Camino, L., Gouveia, L., Maia, L., Paz, M., \& Laureano, M. (2013). Repertórios discursivos contemporâneos sobre as desigualdades raciais no Brasil: Um estudo com estudantes paraibanos de pós-graduação. Psicologia \& Sociedade, 25(1), 113-122. Link

Camino, L., Tavares, T., Torres, A. R., Álvaro, J. L., \& Garrido, A. (2014). Repertórios discursivos de estudantes universitários sobre cotas raciais nas universidades públicas brasileiras. Psicologia \& Sociedade, 26, 117-128. Link

Carvalho, P. (2014). Racismo enquanto teoria e prática social. In J. Jesus, P. Carvalho, R. Diogo \& P. Granjo, $O$ que é racismo? (pp. 37-70). Lisboa: Escolar Editora.

Castro, A., Koelzer, L., Camargo, B., \& Bousfield, A. (2014). Representações sociais na internet sobre cotas para negros em universidades federais. Cadernos de Pesquisa Interdisciplinar em Ciências Humanas, 15(106), 202-220. DOI: $10.5007 / 1984-8951.2014 \mathrm{v} 15 \mathrm{n} 106 \mathrm{p} 202$

Creswell, J. (2007). Projeto de pesquisa: Métodos qualitativo, quantitativo e misto. Porto Alegre: Artmed.

Cruz, I. (2016). Indo muito além do sistema de cotas na Universidade: Quais outras estratégias são necessárias para superação da discriminação institucional? Boletim NEPAE-NESEN, 13(1). Link

Duarte, R. (2008). Políticas de cotas para minorias raciais: uma questão de justiça? Análise do discurso de estudantes da UFPB sobre política de cotas para minorias raciais. Dissertação de Mestrado, Programa de Pós-graduação em Psicologia Social, Universidade Federal da Paraíba, PB.

Faro, A., Silva, P., \& Machado, A. (2016). Como a sociedade os receberá? Preconceito e expectativas de inserção profissional de estudantes egressos do sistema de cotas. Veredas Favip-Revista Eletrônica de Ciências, 8(2), 6-23. Link

Ferreira, R., \& Mattos, R. M. (2007). O afro-brasileiro e o debate sobre o sistema de cotas: Um enfoque psicossocial. Psicologia: Ciência e Profissão, 27(1), 46-63. Link

Freyre, G. (1933/2017). Casa-Grande e Senzala: Formação da família brasileira sob o regime da economia patriarcal. Rio de Janeiro: Maia \& Schmidt.

Guarnieri, F., \& Melo-Silva, L. (2010). Perspectivas de estudantes em situação de vestibular sobre as cotas universitárias. Psicologia \& Sociedade, 22(3), 486-498. Link

Guarnieri, F., \& Melo-Silva, L. (2017). Cotas universitárias no Brasil: Análise de uma década de produção científica. Psicologia Escolar e Educacional, 21(2), 183-193. Link

Instituto Brasileiro de Geografia e Estatística [IBGE]. (2016). Pesquisa nacional por amostra de domicílio: síntese de indicadores 2015. Link

Instituto de Pesquisa Económica Aplicada [IPEA]. (2014). Situação Social da População Negra por Estado. Link

Jesus, J. (2013). O desafio da convivência: Assessoria de diversidade e apoio aos cotistas (2004-2008). Psicologia Ciência e Profissão, 33(1), 222-233. Link 
Lei n. 12.711, de 29 de agosto de 2012 (2012). Dispõe sobre o ingresso nas universidades federais e nas instituições federais de ensino técnico de nível médio e dá outras providências. Link

Lima, M. E. (2002). Normas sociais e racismo: Efeitos do individualismo meritocrático e do igualitarismo na infrahumanização dos negros. Tese de Doutorado, Instituto Universitário de Lisboa, Portugal.

Lima, M. E. (2010). Desigualdades raciais e políticas públicas: Ações afirmativas no governo Lula. Novos Estudos-CEBRAP, (87), 77-95. DOI: 10.1590/S0101-33002010000200005

Lima, M. E. (2013). Preconceito. In L. Camino, A. R. Torres, M. E. Lima \& M. E. Pereira (Eds.), Psicologia social: Temas e teorias (pp. 589-640). Brasília: TechnoPolitk.

Lima, M. E. (2014). Social psychology: Fundamentals and fundamentalisms. Tends in Psychology/ Temas em Psicologia, 22 (1), 179-193. Link

Lima, M. E., Neves, P., \& Silva, P. (2014). A implantação de cotas na universidade: Paternalismo e ameaça à posição dos grupos dominantes. Revista Brasileira de Educação, 19(56), 141-163. Link

Lima, M. E., \& Vala, J. (2004). As novas formas de expressão do preconceito e do racismo. Estudos de Psicologia, 9(3), 401-411. Link

Lima, T. J. S., Pereira, C. R., Torres, A. R. R., Souza, L. E. C., \& Albuquerque, I. M. (2019). Black people are convicted more for being black than for being poor: The role of social norms and cultural prejudice on biased racial judgments. PloS One, 14(9), e0222874. DOI: 10.1371/journal.pone.0222874

Marques, T., \& Santos, B. (2015). Um estudo sobre o pensamento social de jovens universitários acerca da justiça e das cotas raciais. Psicologia e Saber Social, 4(1), 108-125. Link

Mayorga, C., \& Souza, L. (2012). Ação Afirmativa na Universidade: A permanência em foco. Revista psicologia política, 12(24), 263-281. Link

Melo, C., Dantas, G., Fernandez, Z., Pereira, M. E., \& Chaves, A. (2014). Representações sociais de estudantes da UFBA sobre as cotas universitárias. Psicologia e Saber Social, 3(1), 55-69. Link

Mendes, A., Junior. (2014). Uma análise da progressão dos alunos cotistas sob a primeira ação afirmativa brasileira no ensino superior: $\mathrm{O}$ caso da Universidade do Estado do Rio de Janeiro. Avaliação de Políticas Públicas e Educação, 82(22), 31-56. Link

Mendes, K., Silveira, R. C., \& Galvão, C. M. (2008). Revisão integrativa: Método de pesquisa para a incorporação de evidências na saúde e na enfermagem. Texto \& contexto enfermagem, 17(4), 758-764. Link

Munanga, K. (2012). Prefácio. In I. Corone \& M. Bento (Orgs.), Psicologia social do racismo: Estudos sobre branquitude e branqueamento no Brasil (pp. 9-12). Petrópolis: Vozes.

Naiff, D., Naiff, L., \& Souza, M. (2009). As representações sociais de estudantes universitários a respeito das cotas para negros e pardos nas universidades públicas brasileiras. Estudos e Pesquisas em Psicologia, 9(1), 219-232. Link

Oliveira, P., Filho. (2009). A mobilização do discurso da democracia racial no combate às cotas para afrodescendentes. Estudos de Psicologia, 26(4), 429-436. Link

Pereira, C. R., \& Souza, L. (2016). Fatores legitimadores da discriminação: Uma revisão teórica. Psicologia: Teoria e Pesquisa, 32(2), 1-10. DOI: 10.1590/0102-3772e322222

Pereira, C. R., \& Vala, J. (2011). A legitimação da discriminação em diferentes contextos normativos. In E. M. Techio \& 
M. E. Lima (Eds.), Cultura e produção das diferenças: Estereótipos e preconceito no Brasil, Espanha e Portugal (pp. 363-404). Brasília: Technopolitik.

Sacco, A. M., Couto, M., \& Koller, S. H. (2016). Revisão sistemática de estudos da psicologia brasileira sobre preconceito racial. Temas em Psicologia, 24(1), 233-250. DOI: 10.9788/TP2016.1-16

Schulze, C. (2012). Igualdade, discriminação positiva, cotas e ADPF 186. Revista Jus Navigandi, 17(3228). Link

Silva, P., \& Silva, P. (2012). Representação social de estudantes universitários sobre cotas na universidade. Fractal: Revista de Psicologia, 24(3), 525-542. Link

Velloso, J. (2009). Cotistas e não-cotistas: Rendimento de alunos da Universidade de Brasília. Cadernos de Pesquisa, 39(137), 621-644. Link

\section{Como citar:}

Oliveira, I. A., Maia L. M., \& Lima, T. J. S. (2020). Cotas Raciais na Universidade: Uma Revisão Integrativa da Psicologia Brasileira. Revista Subjetividades, 20(Esp 1. Relações Intergrupais: Preconceito e Exclusão Social), e9337. http://doi. org/10.5020/23590777.rs.v20iEsp1.e9337

\section{Endereço para correspondência}

Iara Andrade de Oliveira

E-mail: iara_andrade_@hotmail.com

Luciana Maria Maia Viana

E-mail: lumariamaia@hotmail.com

Tiago Jessé Souza Lima

E-mail: tiago.souzalima@hotmail.com 